\title{
Overexpression of SREBF chaperone (SCAP) enhances nuclear SREBP1 translocation to upregulate fatty acid synthase (FASN) gene expression in bovine mammary epithelial cells
}

\author{
L. Q. Han, ${ }^{*}$ T T. Y. Gao, ${ }^{*}$ G. Y. Yang, ${ }^{* 1}$ and J. J. Loor ${ }^{1}$ \\ ${ }^{*}$ College of Animal Science and Veterinary Medicine, Henan Agricultural University, Zhengzhou 450002, P.R. China \\ †Department of Animal Sciences and Division of Nutritional Sciences, University of Illinois, Urbana 61801
}

\begin{abstract}
Fatty acid synthase is a key enzyme for the synthesis of milk fat in the ruminant mammary gland. In nonruminants, sterol regulatory element binding protein 1 (SREBP1) is a regulator of FASN gene expression, and SREBF chaperone (SCAP) is essential for SREBP1 maturation and activity. However, the role of SCAP on the regulation of $F A S N$ gene expression in ruminants is unknown. The objective of this study was to investigate the transcriptional regulation of FASN by overexpressing SCAP in bovine mammary epithelial cells. A bovine SCAP expression vector, SREBP1 expression vector, and the promoter of $F A S N$ were cloned. The transcription factor binding sites of $F A S N$ promoter were predicted using bioinformatics analysis. After transfection with FASN promoter vectors in the immortalized bovine mammary epithelial cell line MAC-T, we cooverexpressed the SCAP + SREBP1 expression vector with pcDNA3.1 vector as control. The effect of SCAP + SREBP1 overexpression on the regulation of FASN was investigated using luciferase assay, immunofluorescence, Western blot, real-time PCR, and lipid droplet staining. We observed that co-overexpression of SCAP + SREBP1 significantly increased activity of the FASN promoter containing a sterol response element binding site. The FASN mRNA abundance and lipid droplet formation increased due to co-overexpression of SCAP + SREBP1. Compared with overexpression of SREBP1 alone, co-overexpression of SCAP + SREBP1 enhanced the nuclear translocation and nuclear SREBP1 protein abundance. Overall, as in nonruminants cells, results indicate that SCAP is essential for promoting nuclear translocation of SREBP1 and activation of FASN gene
\end{abstract}

\footnotetext{
Received January 3, 2018.

Accepted March 4, 2018.

${ }^{1}$ Corresponding authors: haubiochem@163.com and jloor@illinois. edu
}

transcription, leading to lipid droplet formation in bovine mammary epithelial cells.

Key words: lactation, lipogenesis, milk fat

\section{INTRODUCTION}

The lactating mammary gland is the major lipid-synthesizing organ in the body (Rudolph et al., 2010). Fatty acid synthase (FASN), as a key metabolic enzyme that catalyzes the synthesis of long-chain fatty acids from acetyl-CoA and malonyl-CoA, is essential for functional development of mammary gland and milk fat production (Suburu et al., 2014). The bovine FASN gene is mapped to chromosome 19 and several SNP in the $5^{\prime}$ flanking region are significantly associated with milk fat content in dairy cattle (Roy et al., 2006; Ordovás et al., 2008). Therefore, studying the regulation of FASN will enhance mechanistic knowledge and may provide tools for practical manipulation of milk fat synthesis.

The regulation of $F A S N$ is primarily dependent on transcription factors that bind to the specific cis-acting elements located on the promoter (Griffin et al., 2007). Several potential transcriptional regulatory elements have been identified in the $5^{\prime}$ flanking region of rat and human FASN promoter that mediate both positive and negative effects on its abundance (Hsu et al., 1996; Oskouian et al., 1997).

Sterol regulatory element binding protein 1 (SREBP1) is a key transcription factor whose transcriptional activation coincides with copious milk fat synthesis at the onset and throughout lactation in dairy cows (Bionaz and Loor, 2008; Loor et al., 2013). The SREBP1 are synthesized as inactive precursors in the endoplasmic reticulum (ER) and form complexes with SREBF chaperone (SCAP; an ER-Golgi transporter). In the Golgi apparatus, SREBP1 are hydrolyzed by proteases and the transcriptional fragments of nuclear SREBP1 released to activate lipogenic genes (Moon et al., 2012; Moon, 2017). In vitro work with the nonsecretory MAC-T cell line indicated that abundance of FASN could be regulated by SREBP (Ma and Corl, 
2012). In fact, the role of SREBP in lipid synthesis has been well established in bovine mammary epithelial cells ( $\mathrm{Li}$ et al., 2014).

At least in nonruminants, the SCAP protein is essential for the activation of SREBP, and in its absence the protease fails to cleave SREBP and prevents the transcriptionally active fragments of SREBP from entering the cell nucleus to activate gene expression (Rawson et al., 1999). Inhibition of SCAP function reduced SREBP-regulated gene expression and de novo lipogenesis in rhesus monkeys (Jensen et al., 2016). In ob/ob mice, knockdown of the hepatic SCAP gene reduced nuclear SREBP expression and produced a dramatic decrease in fatty acid (FA) synthesis (Moon, 2017). However, little is known about the role of SCAP in the transcriptional regulation of FASN abundance in ruminant mammary cells.

We hypothesized that SCAP might enhance SREBP1 activation of $F A S N$ gene abundance. To investigate this hypothesis, we constructed a bovine SREBP1 vector, a SCAP vector, and a $F A S N$ promoter vector and assessed the effect of overexpression of SCAP on the FASN promoter activity, mRNA expression, and lipid droplet formation in bovine mammary epithelial cells.

\section{MATERIALS AND METHODS}

\section{Plasmid Construction}

Total RNA was extracted from bovine mammary tissue samples using TRIzol reagent (15596, Invitrogen, Carlsbad, CA) and was reverse-transcribed to cDNA as a template for PCR. To construct the SCAP and SREBP1 expression plasmid, full-length open reading frame sequences were amplified from cDNA using PCR and subcloned into the restriction sites of the
pcDNA3.1 $(+)$ vector. The PCR primer pair of SCAP and SREBP1 encoded His-tag and Myc-tag protein sequences at the $\mathrm{N}$ terminus, respectively (primers are listed in Table 1).

\section{FASN Promoter Reporter Construction and Characterization}

Bovine genomic DNA was extracted from blood and used as a template for amplifying the $F A S N$ gene promoter. Three different 5 ' flanking fragments of various lengths containing FASN promoter region (GenBank Accession Number: AF285607) were amplified by PCR. Promoters of 399, 255, and 177 bp were produced using PCR primers designed to hybridize at the corresponding positions and used with the common downstream primer (primers are listed in Table 1). The PCR fragment of FASN promoter was gel purified and digested with $K p n I$ and HindIII restriction enzymes to clone into the pGL3-Basic Vectors (E1741, Promega, Madison, WI). Plasmid DNA from selected clones was identified by restriction enzyme and then sequenced. The potential transcription factor binding sites were predicted using online bioinformatics software (Roy et al., 2005; Li et al., 2015). The sterol response element (SRE) sequence of the bovine FASN promoter was aligned to that of human (GenBank: AF250144), rat (GenBank: X54671), and goat (GenBank: kp749922) using the BioXM2.6 software (Institute of Rice Research, Nanjing Agricultural University, Nanjing, China).

\section{Cell Culture and Transient Transfection}

Experiments were performed using the immortalized bovine mammary epithelial cell line MAC-T. The MAC$\mathrm{T}$ cell line was produced from primary bovine mammary

Table 1. Primers used for cloning the open reading frame of SREBP1, open reading frame of SCAP, the FASN promoter and FASN mRNA

\begin{tabular}{|c|c|c|}
\hline $\begin{array}{l}\text { Gene symbol and GenBank } \\
\text { accession number }\end{array}$ & Primer $^{1}$ sequence $\left(5^{\prime}-3^{\prime}\right)$ & $\begin{array}{c}\text { Product } \\
\text { length } \\
\text { (bp) }\end{array}$ \\
\hline $\begin{array}{l}S C A P \\
\text { NM_001101889 } \\
S R E B P 1 \\
\text { NM_001113302.1 } \\
\text { FASN }{ }^{2} \\
\text { AF285607 }\end{array}$ & $\begin{array}{l}\text { F: CCCAAGCTTATGCATCATCACCATCACCATACCCTGACTGAAAG } \\
\text { R: ATTTGCGGCCGCTCAGTCCAGCTTCTCCA } \\
\text { F: CTAGCTAGCATGGAACAAAAACTCATCTCAGAAGAGGATCTGGACGAGCC } \\
\text { R: TGCTCTAGACTAGCTGGAGGTCACAGTGGTCCCA } \\
F A S N \text { 3F: CGGGGTACCGGGAGGCGTGGAGCACGGAA } \\
F A S N \text { 2F: CGGGGTACCGCATCACCCCACTGGCGGC } \\
F A S N \text { 1F: CGGGGTACCCTGTCAGCCCATGTGGCGTGTC } \\
F A S N \text { R: CCCAAGCTTTGGCCGCTTGTACCTGGTCTGG } \\
\text { Q F: ACCTCGTGAAGGCTGTGACTCA } \\
\text { Q R: TGAGTCGAGGCCAAGGTCTGAA }\end{array}$ & $\begin{array}{r}3,876 \\
3,486 \\
399 \\
255 \\
177 \\
92\end{array}$ \\
\hline
\end{tabular}


alveolar cells by stable transfection with SV-40 large T-antigen (Huynh et al., 1991). Cells were cultured in basal Dulbecco's modified Eagle's medium containing $100 \mathrm{U} / \mathrm{mL}$ of penicillin, $100 \mu \mathrm{g} / \mathrm{mL}$ of streptomycin, 5 $\mu \mathrm{g} / \mathrm{mL}$ of insulin, $1 \mu \mathrm{g} / \mathrm{mL}$ pf hydrocortisone, and $10 \%$ fetal bovine serum. The MAC-T cells were cultured in a lactogenic medium (Kadegowda et al., 2009). The cells were counted and seeded at a density of $5 \times 10^{5}$ in 6 -well plates and then cultured until 70 to $80 \%$ confluence before transfection with FASN promoter, SCAP vector, or SREBP1 vector using Lipofectamine 3000 Transfection Reagent (L3000008, Invitrogen) according to the manufacturer's protocol (https://www.thermofisher.com/ us/en/home/brands/product-brand/lipofectamine/ lipofectamine-3000.html). Briefly, the vector DNA and Lipofectamine 3000 reagent mixture was prepared by diluting in Opti MEM medium (Gibco, Grand Island, NY) followed by thorough mixing. The mixture was incubated for $15 \mathrm{~min}$ at room temperature before addition of the DNA-lipid complex to the MAC-T cells.

\section{Promoter Luciferase Assay}

The promoter activity in cell lysate was measured in a luminometer (Fluroskan Ascent FL, Thermo Fisher Scientific, Waltham, MA) using the Dual-luciferase Reporter Assay System (E1910, Promega). Briefly, after $48 \mathrm{~h}$ of transfection, MAC-T cells were washed twice with PBS and then lysed with the passive lysis buffer for $15 \mathrm{~min}$ at room temperature. Twenty microliters of lysate were transferred to a microcentrifuge tube and $100 \mu \mathrm{L}$ of Luciferase Assay Reagent II (Promega) was added before measuring firefly luciferase activity; 100 $\mu \mathrm{L}$ of stop reagent was then added, followed by measuring Renilla luciferase activity. The relative luciferase activity of the promoter was calculated using the ratio of firefly luciferase activity/Renilla luciferase activity.

\section{Real-Time PCR}

Total RNA was extracted from MAC-T cells using TRIzol Plus RNA Purification Kit (12183555, Thermo Fisher Scientific) according to the manufacturer's protocol (https://www.thermofisher.com/order/catalog/ product/12183555?SID=srch-hj-12183555). The firststrand cDNA was synthesized using a Prime Script RT Reagent kit with gDNA Eraser (TaKaRa, Shiga, Japan). Quantitative real-time PCR analysis was performed using SYBR fast quantitative real-time PCR mix (TaKaRa) in a 7500 fast Real-Time PCR Systems (Thermo Fisher Scientific). The FASN mRNA abundance was measured by quantitative PCR (primers are listed in Table 1). The data were analyzed using the standard curve method and the geometric mean of the internal control genes UXT, RPS15, and RPS9 (Bionaz and Loor, 2007).

\section{Lipid Droplet Staining}

After transfection, MAC-T cells were washed twice with PBS for 3 min, fixed with $4 \%$ paraformaldehyde for $20 \mathrm{~min}$, and then gently washed 3 times with PBS. Nile red $(10 \mu \mathrm{g} / \mathrm{mL})$ was added to each well and cells were incubated for $15 \mathrm{~min}$ at room temperature. Cells were then washed with PBS for 3 times in the dark, followed by measuring fluorescence intensity at excitation wavelength of $480 \mathrm{~nm}$ and emission wavelength of $575 \mathrm{~nm}$ in a full-wavelength microplate reader (Varioskan LUX, Thermo Fisher Scientific). To stain nuclei after Nile red, $100 \mu \mathrm{g} / \mathrm{mL}$ of DAPI (4',6-diamidino2-phenylindole) were added followed by incubation for 5 min. Cells were then washed 3 times with PBS and fluorescence determined using an inverted microscope (IX73, Olympus, Tokyo, Japan).

\section{Immunofluorescence of Nuclear SREBP1 Protein}

The MAC-T cells were plated onto Lys-coated slides and used for immunostaining after transfection with vectors. Cells were washed 3 times with PBS and then fixed with $4 \%$ paraformaldehyde for $20 \mathrm{~min}$. After washing 3 more times, cells were incubated with blocking solution (90\% PBS $+10 \%$ bovine serum) for $1 \mathrm{~h}$ and then incubated with mouse monoclonal anti-Myc tag antibody (9E10; 1:500, ab32, Abcam, Cambridge, UK) overnight at $4^{\circ} \mathrm{C}$. Cells were washed 3 times with PBS and incubated with Alexa Fluor 488 Donkey AntiMouse $\operatorname{IgG}\left(\mathrm{H}^{+} \mathrm{L} ; 1: 1,000\right.$, R37114, Invitrogen) for 1 h. After washing 3 times and covering with a glass coverslip, the image was collected with a laser confocal microscope (LSM 5 PASCAL, Zeiss, Oberkochen, Germany). The nuclear fluorescence intensity of SREBP1 was quantified by using Image $\mathrm{J}$ software (National Institutes for Health, Bethesda, MD).

\section{Western Blot}

Protein was extracted from transfected cells using RIPA lysis and extraction buffer (89900, Thermo Fisher Scientific). Protein concentration was measured using the Pierce BCA assay kit (23225, Thermo Fisher Scientific). Fifty micrograms of total protein per lane was loaded on SDS-PAGE and transferred to a polyvinylidene difluoride membrane. After blocking with $5 \%$ skim milk for $1 \mathrm{~h}$, the membranes were incubated with primary antibody at $4^{\circ} \mathrm{C}$ overnight. The primary antibodies used in this study were rabbit polyclonal of anti- $\beta$-actin (1:1000, bs-0061R, Bioss, Woburn, MA), 
anti-SREBP1 (1:500, NB100-2215, Novusbio, Littleton, CO), and anti-SCAP (1:1000, ab190103, Abcam). After washing 5 times with Tris-buffered saline-Tween, the membrane was probed with HRP-conjugated Affinipure Goat Anti-Rabbit IgG( $\mathrm{H}^{+} \mathrm{L} ; 1: 2,000$, SA00001-2, Proteintech, Rosemont, IL) for $1 \mathrm{~h}$ at room temperature. Quantification of immunoblots was performed by a standard enhanced chemiluminescence procedure (Batistel et al., 2017).

\section{Statistical Analysis}

All the treatments were run in triplicate and results are reported as mean \pm standard error of the means. The data were analyzed using SPSS 19.0 (SPSS Inc., Chicago, IL). Statistical analysis was performed using Student's $t$-test (unpaired and 2-tailed) with 2 groups. A 1-way ANOVA was used for multiple comparisons followed by Tukey's test. Differences between 2 treatments were considered statistically significant at $P<$ 0.05 .

\section{RESULTS}

\section{Characterization of the 5' Flanking Sequence of Bovine FASN Gene}

Bioinformatics analysis revealed that $399 \mathrm{bp}$ of the $5^{\prime}$ flanking FASN sequence contains several binding sites for transcription factors (Figure 1A), including specificity protein 1 (Sp1), CCAAT-enhancer-binding protein (C/EBP), nuclear factor Y (NF-Y), and early growth response protein 1 (EGR-1). It also contains an SRE (5'-ATCACCCCAC-3') with high similarity relative to the SRE region of human, rat, and goat (Figure 1B).

\section{Basal FASN Promoter Activity}

The fragments designed to include different specific regions in the FASN promoter sequence were pGL3FASN1 (177 bp) mainly containing a TATA-box (Figure 2A), pGL3-FASN2 (255 bp) extended to mainly contain the SRE domain, and pGL3-FASN3 (399 bp)

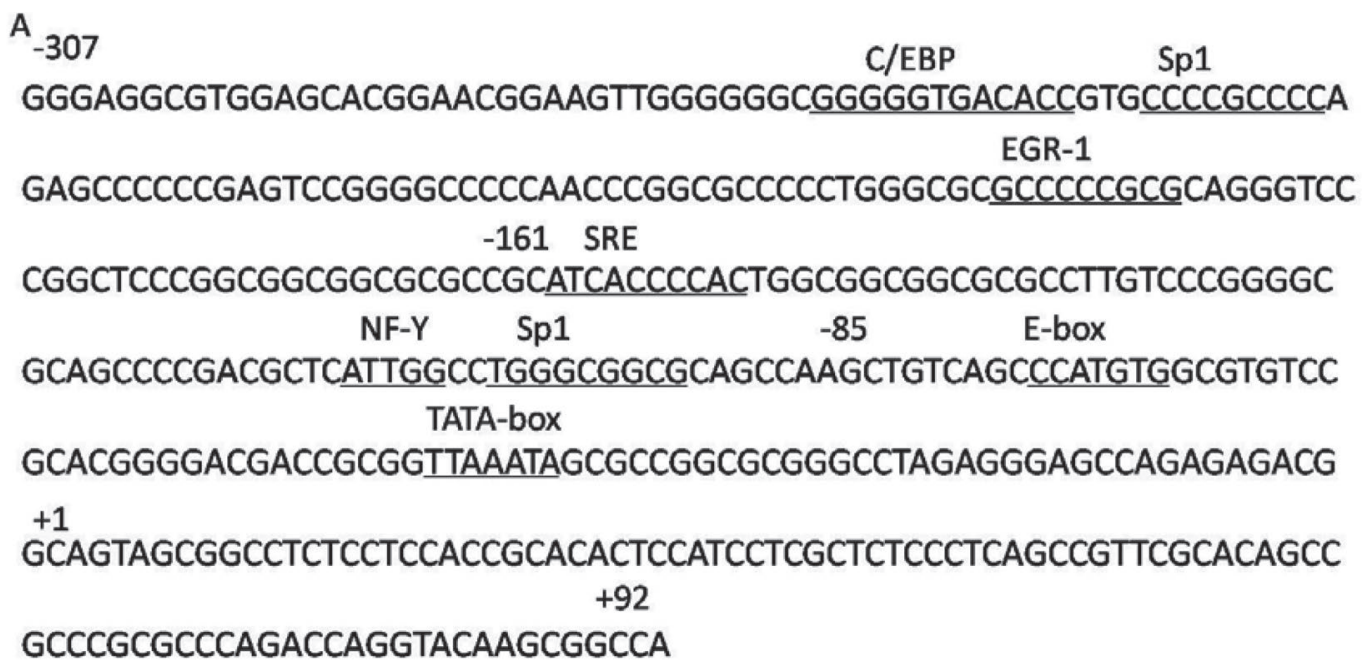

B

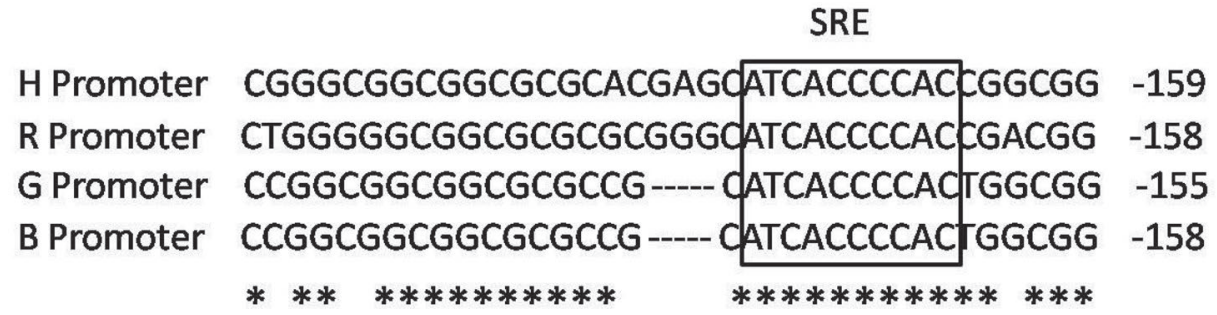

Figure 1. Bioinformatics analysis of the bovine FASN promoter region. (A) Sequence analysis of the bovine $5^{\prime} F A S N$ flanking sequence. The transcription initiation site is designated as +1 , where + or - indicate the number of bases downstream or upstream from the transcription start site. The binding site sequences for transcription factors are underlined, and the name of transcription factor are indicated above the sequence. (B) Alignment of sterol response element (SRE) binding site sequences of the human, rat, goat, and bovine FASN gene using BioXM 2.6 (Institute of Rice Research, Nanjing Agricultural University, Nanjing, China). *Indicates similarity across all sequences. C/EBP $=$ CCAATenhancer-binding protein; Sp1 = specificity protein 1; EGR-1 = early growth response protein 1; NF-Y = nuclear factor Y; E-box $=$ enhancer box. 
A pGL3-FASN3 399bp
pGL3-FASN2 255bp
pGL3-FASN1 177bp

TATA-box:TTAAATA

SRE binding site: ATCACCCCAC

C/EBP :GGGGGTGACACC

B

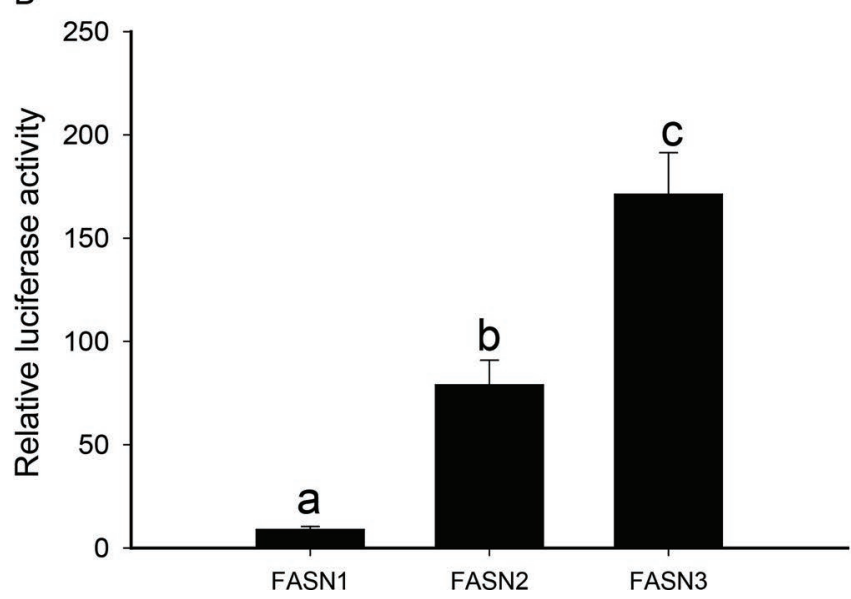

Figure 2. Bovine $F A S N$ promoter reporter construction and basal transcriptional activity. (A) Different bovine FASN promoter fragments (pGL-FASN1 3) were cloned into the pGL3-basic luciferase reporter vector (LUC). The potential binding site regions for important transcription factors $(\mathrm{SRE}=$ sterol response element; $\mathrm{C} / \mathrm{EBP}=$ CCAAT-enhancer-binding protein) are indicated along with promoter fragment sizes. (B) $F A S N$ promoters (pGL3-FASN1, pGL3- FASN2, pGL3-FASN3) were transiently transfected into the bovine mammary epithelial cell line MAC-T. The relative luciferase activity of the promoter was analyzed using the ratio of firefly luciferase activity/Renilla luciferase activity. Different letters $(\mathrm{a}-\mathrm{c})$ indicate means differ at $P<$ 0.05. Color version available online.

extended $144 \mathrm{bp}$ further than pGL3-FASN2 to mainly contain the $\mathrm{C} / \mathrm{EBP}$ domain. The basal promoter activity of pGL3-FASN1 3 is shown in Figure 2B. Whereas the short 155bp pGL3-FASN1 had basal transcriptional activity, pGL3-FASN2 had 10-fold greater promoter activity. In addition, a further 2-fold increase of activity was observed with the pGL3-FASN3 promoter.

\section{Overexpression of SCAP Increased SREBP1- Regulated FASN Promoter Activity}

Compared with pcDNA3.1, overexpression of SREBP1 significantly activated FASN2 and FASN3 promoter activity $(P<0.05)$, whereas overexpression of SCAP had no effect (Figure 3A). Compared with overexpression SREBP1, co-overexpression of SCAP + SREBP1 increased FASN2 and FASN3 promoter activity $(P<0.05$, Figure $3 \mathrm{~A})$. Compared with pcDNA3.1, increasing the overexpression dose of SCAP led to significant increase in FASN2 promoter activity $(P<0.05$, Figure 3B).
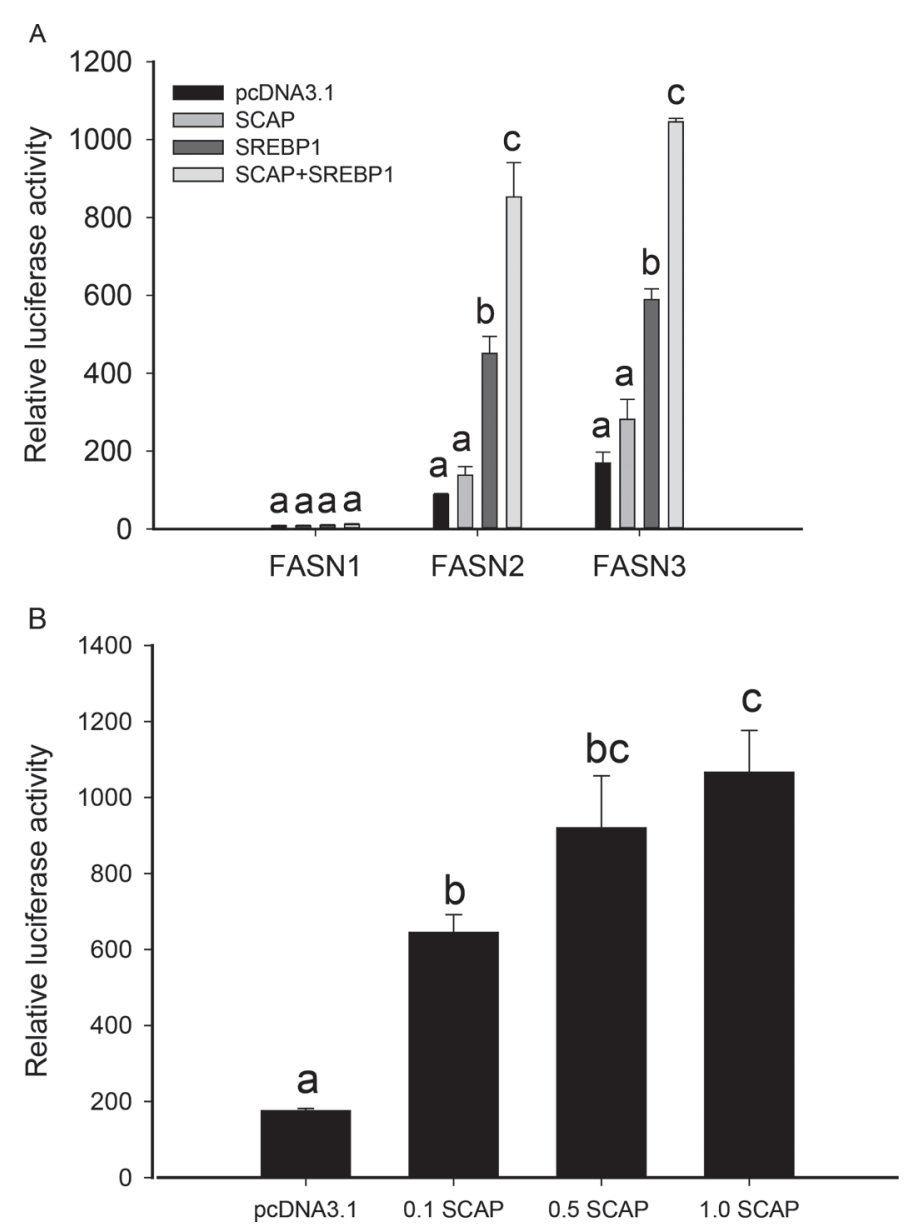

Figure 3. Effect of overexpression of SREBF chaperone (SCAP) on FASN promoter activity in the bovine mammary epithelial cell line MAC-T. (A) Cells were transiently transfected with the pGL$F A S N 1 \sim 3$ reporter vector, SCAP, or sterol regulatory element binding protein 1 (SREBP1) vector. Treatments were pcDNA3.1 $(2 \mu \mathrm{g}$ of pcDNA3.1 empty vector), SCAP (1 $\mathrm{gg}$ of pcDNA3.1 $+1 \mu \mathrm{g}$ of pcDNA3.1-SCAP), SREBP1 (1 $\mu \mathrm{g}$ of pcDNA3.1 + $1 \mu \mathrm{g}$ of pcDNA3.1SREBP1), or SCAP + SREBP1 (1 of $\mu \mathrm{g}$ pcDNA3.1-SREBP1 + 1 $\mu \mathrm{g}$ of pcDNA3.1-SCAP) vector. SCAP + SREBP1 denotes the cooverexpression of SCAP and SREBP1. Cell extracts were assayed for luciferase activity. (B) Cells were transfected with the pGL-FASN2 reporter vector, SCAP, or SREBP1 vector. Treatments were pcDNA3.1 $(2 \mu \mathrm{g}$ of pcDNA3.1 empty vector), 0.1 SCAP $(0.9 \mu \mathrm{g}$ of pcDNA3.1 $+0.1 \mu \mathrm{g}$ of pcDNA3.1-SCAP $+1 \mu \mathrm{g}$ of pcDNA3.1-SREBP1), 0.5 $\mathrm{SCAP}(0.5 \mu \mathrm{g}$ of pcDNA3.1 $+0.5 \mu \mathrm{g}$ of pcDNA3.1-SCAP $+1 \mu \mathrm{g}$ of pcDNA3.1-SREBP1), 1.0 SCAP $(1.0 \mu \mathrm{g}$ of pcDNA3.1-SCAP $+1.0 \mu \mathrm{g}$ of pcDNA3.1-SREBP1). Different letters $(\mathrm{a}-\mathrm{c})$ indicate means differ at $P<0.05$. 


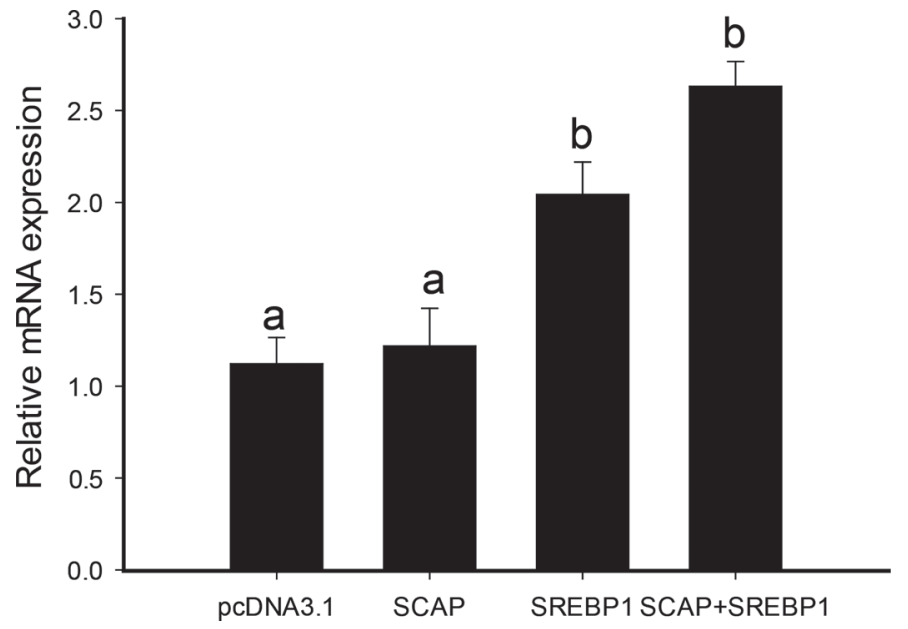

Figure 4. Effect of overexpression of SREBF chaperone (SCAP) on FASN mRNA abundance in the bovine mammary epithelial cell line MAC-T. Cells were transiently transfected with pcDNA3.1 vector, SCAP, or sterol regulatory element binding protein 1 (SREBP1) vector. The treatment groups were pcDNA3.1 ( $2 \mu \mathrm{g}$ of pcDNA3.1 empty vector), SCAP (1 $\mu \mathrm{g}$ of pcDNA3.1 $+1 \mu \mathrm{g}$ of pcDNA3.1-SCAP), SREBP1 (1 $\mu \mathrm{g}$ of pcDNA3.1 + $1 \mu \mathrm{g}$ of pcDNA3.1-SREBP1), or SCAP + SREBP1 $(1 \mu \mathrm{g}$ of pcDNA3.1-SREBP1 $+1 \mu \mathrm{g}$ of pcDNA3.1-SCAP) vector. SCAP + SREBP1 denotes the co-overexpression of SCAP and SREBP1. Total RNA was extracted from cells and used for measuring FASN mRNA expression by quantitative PCR. Different letters $(\mathrm{a}, \mathrm{b})$ indicate means differ at $P<0.05$.

\section{Overexpression of SCAP Upregulated FASN mRNA and Increased Lipid Droplet Formation}

Compared with pcDNA3.1, overexpression of SREBP1 and co-overexpression SCAP + SREBP1 markedly upregulated FASN mRNA abundance (Figure 4) and increased lipid droplet relative fluorescence intensity (Figure 5A, 5B). Overexpression of SCAP had no effect on FASN mRNA abundance and lipid droplet formation. Compared with overexpression SREBP1, co-overexpression of SCAP + SREBP1 significantly increased lipid droplet fluorescence (Figure 5B).

\section{Overexpression of SCAP Promoted Nuclear Translocation of SREBP1}

Overexpression of SCAP promoted nuclear translocation of SREBP1 (Figure 6A) and increased nuclear fluorescence intensity of SREBP1 $(P<0.0001$, Figure $6 \mathrm{~B})$. Overexpression of SCAP led to increased levels of SCAP protein and nuclear SREBP1 protein $(P<0.01$, Figure 6C, 6D).

\section{DISCUSSION}

Evidence to date from in vitro and in vivo studies indicates that SREBP1 plays a key role in the regulation of milk fat synthesis in dairy cows (Osorio et

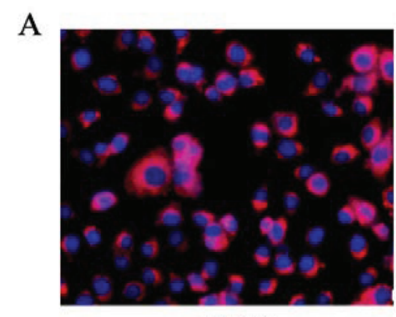

pCDNA3.1

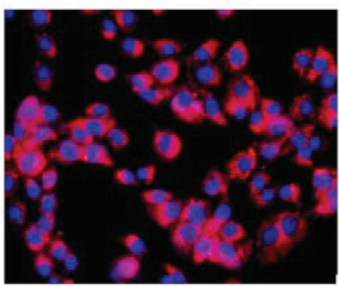

SREBP1

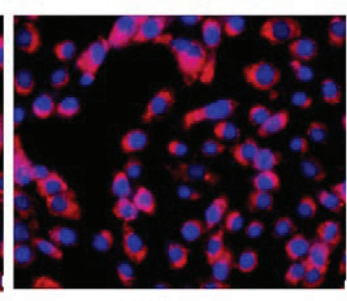

SCAP

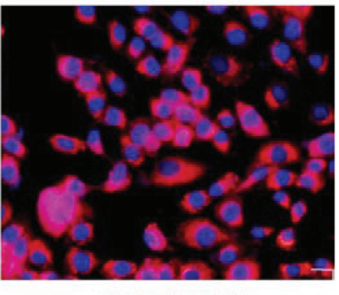

SCAP+SREBP1
B

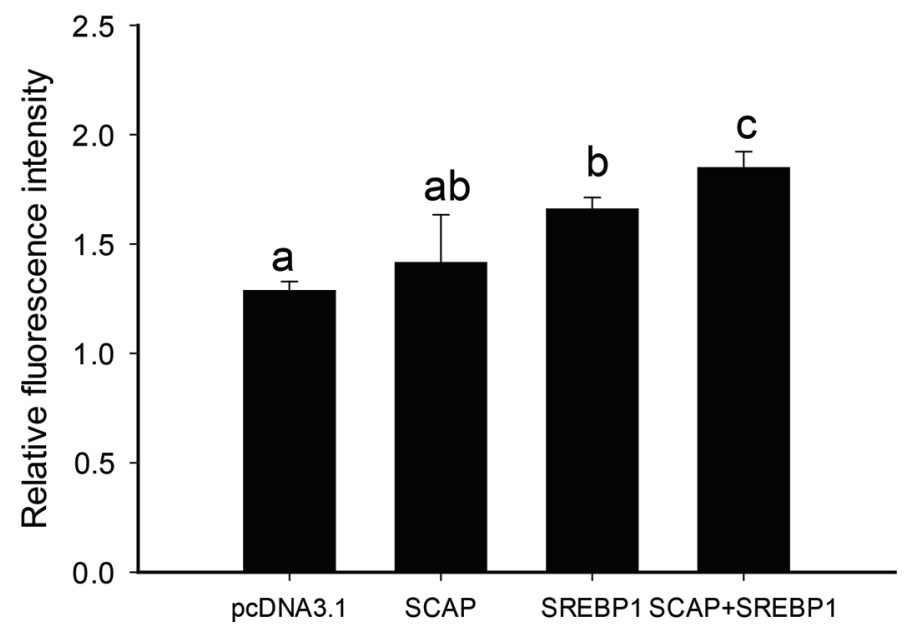

Figure 5. Effect of overexpression of SREBF chaperone (SCAP) on lipid droplet formation in the bovine mammary epithelial cell line MAC-T. Cells were transiently transfected with pcDNA3.1vector, SCAP, or sterol regulatory element binding protein 1 (SREBP1) vector. The treatment groups were pcDNA3.1 (2 $\mu \mathrm{g}$ of pcDNA3.1 empty vector), SCAP (1 $\mathrm{Sg}$ of pcDNA3.1 + $1 \mu \mathrm{g}$ of pcDNA3.1-SCAP), SREBP1 $(1 \mu \mathrm{g}$ of pcDNA3.1 + $1 \mu \mathrm{g}$ of pcDNA3.1-SREBP1), or SCAP + SREBP1 $1 \mu \mathrm{g}$ of pcDNA3.1-SREBP1 + $1 \mu \mathrm{g}$ of pcDNA3.1-SCAP) vector. SCAP + SREBP1 denotes the co-overexpression of SCAP and SREBP1. (A) Lipid droplet was staining with Nile red, nuclei were stained with DAPI (blue); scale bar $30 \mu \mathrm{m}$. (B) Relative fluorescence intensity of lipid droplets was quantified at excitation wavelength of $480 \mathrm{~nm}$ and emission wavelength of $575 \mathrm{~nm}$ in a full-wavelength microplate reader. Different letters $(\mathrm{a}-\mathrm{c})$ indicate means differ at $P<0.05$. Color version available online.

al., 2016) and goats (Carcangiu et al., 2013; Xu et al., 2016a, 2018; Yao et al., 2017). Thousands of target genes regulated by SREBP1 have been identified in human and mouse genomes via CHIP-chip and CHIP-seq technology, including FASN and other lipogenic genes (Reed et al., 2008; Seo et al., 2009). Despite the amount of data available, the regulatory mechanism of FASN 
A

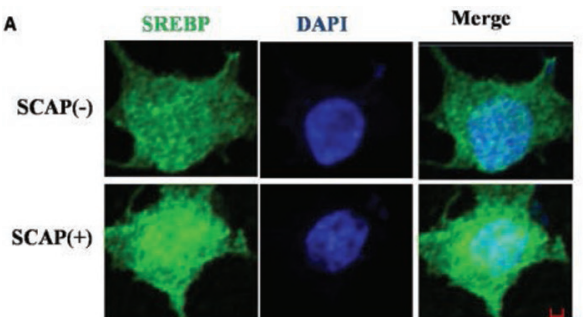

B

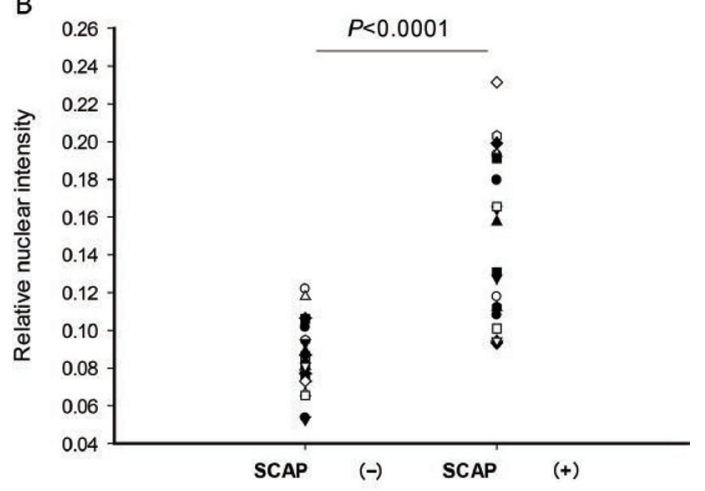

C

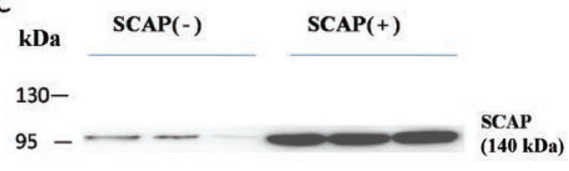

$72-$

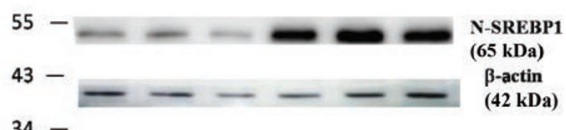

$34-$

D

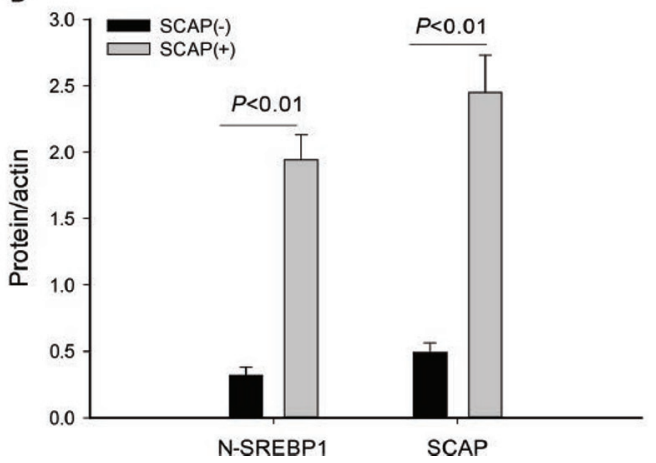

Figure 6. Effect of overexpression of SREBF chaperone (SCAP) on nuclear sterol regulatory element binding protein 1 (SREBP1) expression in the bovine mammary epithelial cell line MAC-T. Cells were transiently transfected with pcDNA3.1 vector, SCAP, or SREBP1 vector. Treatments were $\operatorname{SCAP}(-)(1 \mu \mathrm{g}$ of pcDNA3.1 $+1 \mu \mathrm{g}$ of pcDNA3.1-SREBP1), or $\operatorname{SCAP}(+)(1 \mu \mathrm{g}$ of pcDNA3.1-SCAP +1 $\mu \mathrm{g}$ of pcDNA3.1-SREBP1). (A) Confocal microscopy imaging demonstrating subcellular localization of bovine SREBP1 (green), nuclei were stained with DAPI (blue); scale bar 3,000 nm. (B) Relative nuclear fluorescence intensity of SREBP1 quantified over 20 cells using ImageJ software (National Institutes of Health, Bethesda, MD). (C) Expression level of proteins assessed by Western blot; $\mathrm{N}$ denotes the mature nuclear form of SREBP1. (D) Relative fold quantification of protein of interest relative to $\beta$-actin (grayscale scan). Color version available online. transcription still is not well understood in ruminant cells. As in nonruminants, our study demonstrates that overexpression of bovine SCAP promotes SREBP1 nuclear translocation and leads to increased FASN promoter activity, FASN mRNA abundance, and lipid droplet formation in MAC-T cells.

The finding that the $5^{\prime}$ frank sequence of bovine $F A S N$ harbors potential regulatory elements (Sp1, NF-Y) is in agreement with results from humans, rats, and goats (Roy et al., 2005; Li et al., 2015). Alignment of human, rat, goat, and bovine FASN promoter sequences suggests that this -150 SRE region is highly conserved among species. In mice and humans, this SRE region was reported to be important for the response of SREBP (Moon et al., 2002; Griffin et al., 2007). The SREBP isoforms have a tyrosine residue that allows direct binding to SRE (5'TCACNCCAC-3'; Rome et al., 2008). The transcription factors Sp1 and NF-Y have been shown to function as co-regulators in SREBP-responsive promoters (Magaña et al., 2000; Chakravarty et al., 2004). Therefore, it is not surprising that the constructed FASN2 and FASN3 promoters, which contain SRE and both Sp1 and NF-Y binding sites, had a higher basic promoter activity than FASN1.

In $3 \mathrm{~T} 3-\mathrm{L} 1$ fibroblasts, the rat $F A S N$ promoter is activated by SREBP via the SRE located at -150 (Latasa et al., 2000). In the present study, the increased FASN2 and FASN3 promoter activity due to overexpression of SREBP1 underscores that the SRE region is important for the response of bovine SREBP1 (i.e., SREBP can bind to the $-150 \mathrm{SRE}$ site to activate $F A S N$ promoter). Co-overexpression of SCAP + SREBP1 and overexpression of SCAP in a dose-dependent manner also confirmed that SCAP enhances the FASN promoter activity via SREBP1.

It has been demonstrated that $F A S N$ gene expression is associated with cellular lipid droplet formation in goat mammary epithelial cells (Zhu et al., 2015), and overexpression of SREBP increased the mRNA abundance of FASN and triacylglycerol secretion in bovine and goat mammary epithelial cells (Li et al., 2014; Xu et al., 2016b). Those responses were confirmed in the present study and extended to demonstrate the role of SCAP in the SREBP1 activation of FASN and lipid droplet formation. Transcriptional regulation of milk fat synthesis during lactation in dairy cows involves the coordinated transcription not only of FASN, but also various target lipogenic genes culminating in the formation of lipid droplets (Bionaz and Loor, 2008; Li et al., 2014). Therefore, the present data not only confirmed the cooperative nature of SCAP and SREBP in the regulation of FASN, but its feed-forward effect on lipid droplet formation in bovine mammary epithelial cells. 
The fact that activation of SREBP relies on SCAPescorted trafficking from ER to the Golgi in nonruminants is well known, especially in liver cells (Gong et al., 2006). These events are closely associated with intracellular sterol levels, and once their concentrations decrease, SCAP escorts SREBP to the Golgi apparatus where further processing generates a nuclear form of SREBP1 (N-terminal) that can travel to the nucleus to activate target genes (Sato, 2009). In nonruminant cells, overexpression of the transmembrane domain of SCAP eliminated the ability of sterols to retain the SCAP-SREBP complex in ER (Yang et al., 2000). Our data demonstrating that overexpression of SCAP increased nuclear fluorescence intensity of SREBP1 and protein abundance of mature SREBP1 is evidence that it promoted SREBP1 translocation to the nucleus as in nonruminant cells. The end result of these mechanisms is upregulation of FASN mRNA abundance accompanied by increased lipid droplet accumulation. To our knowledge, these are the first data confirming that SCAP regulates translocation of mature SREBP1 to control transcription of a key lipogenic gene, and the subsequent synthesis of lipid droplets in bovine mammary epithelial cells.

\section{CONCLUSIONS}

As in nonruminant cells, SCAP is essential for promoting nuclear translocation of SREBP and the ensuing lipogenic response through the upregulation of FASN mRNA abundance. Further understanding of the regulatory role of SCAP on the interactive networks among transcription factors involved in coordination of milk fat synthesis in bovine mammary epithelial cells will contribute tools for manipulating milk fat composition in dairy cows.

\section{ACKNOWLEDGMENTS}

This research was jointly supported by the Transgenic New Species Breeding Program of China (Beijing, China; 2016ZX08006), China Postdoctoral Science Foundation (Beijing, China; 166947), and Natural Science Foundation of Henan Province (Zhengzhou, China; 162300410152). L. Q. Han was recipient of a visiting scholarship from China Scholarship Council (Beijing) to train at University of Illinois. The authors declare no competing financial interests.

\section{REFERENCES}

Batistel, F., A. S. Alharthi, L. Wang, C. Parys, Y. X. Pan, F. C. Cardoso, and J. J. Loor. 2017. Placentome nutrient transporters and mammalian target of rapamycin signaling proteins are altered by the methionine supply during late gestation in dairy cows and are associated with newborn birth weight. J. Nutr. 147:1640-1647. https://doi.org/10.3945/jn.117.251876.

Bionaz, M., and J. J. Loor. 2007. Identification of reference genes for quantitative real-time PCR in the bovine mammary gland during the lactation cycle. Physiol. Genomics 29:312-319. https://doi .org/10.1152/physiolgenomics.00223.2006.

Bionaz, M., and J. J. Loor. 2008. Gene networks driving bovine milk fat synthesis during the lactation cycle. BMC Genomics 9:366. https://doi.org/10.1186/1471-2164-9-366.

Carcangiu, V., M. C. Mura, C. Daga, S. Luridiana, S. Bodano, G. A. Sanna, M. L. Diaz, and G. Cosso. 2013. Association between SREBP-1 gene expression in mammary gland and milk fat yield in Sarda breed sheep. Meta Gene 1:43-49. https://doi.org/10.1016/j .mgene.2013.10.001.

Chakravarty, K., S. Y. Wu, C. M. Chiang, D. Samols, and R. W. Hanson. 2004. SREBP-1c and Sp1 interact to regulate transcription of the gene for phosphoenolpyruvate carboxykinase (GTP) in the liver. J. Biol. Chem. 279:15385-15395. https://doi.org/10.1074/ jbc.M309905200.

Gong, Y., J. N. Lee, P. C. Lee, J. L. Goldstein, M. S. Brown, and J. Ye. 2006. Sterol-regulated ubiquitination and degradation of Insig-1 creates a convergent mechanism for feedback control of cholesterol synthesis and uptake. Cell Metab. 3:15-24. https://doi .org/10.1016/j.cmet.2005.11.014.

Griffin, M. J., R. H. Wong, N. Pandya, and H. S. Sul. 2007. Direct interaction between USF and SREBP-1c mediates synergistic activation of the fatty-acid synthase promoter. J. Biol. Chem. 282:54535467. https://doi.org/10.1074/jbc.M610566200.

Hsu, M. H., S. S. Chirala, and S. J. Wakil. 1996. Human fatty-acid synthase gene. Evidence for the presence of two promoters and their functional interaction. J. Biol. Chem. 271:13584-13592.

Huynh, H. T., G. Robitaille, and J. D. Turner. 1991. Establishment of bovine mammary epithelial cells (MAC-T): An in vitro model for bovine lactation. Exp. Cell Res. 197:191-199.

Jensen, K. K., M. Tadin-Strapps, S. P. Wang, J. Hubert, Y. Kan, Y. Ma, D. G. McLaren, S. F. Previs, K. B. Herath, A. Mahsut, A. Liaw, S. Wang, S. J. Stout, C. Keohan, G. Forrest, D. Coelho, S. Yendluri, S. Williams, M. Koser, S. Bartz, K. O. Akinsanya, and S. Pinto. 2016. Dose-dependent effects of siRNA-mediated inhibition of SCAP on PCSK9, LDLR, and plasma lipids in mouse and rhesus monkey. J. Lipid Res. 57:2150-2162. https://doi.org/10.1194/ jlr.M071498.

Kadegowda, A. K., M. Bionaz, L. S. Piperova, R. A. Erdman, and J. J. Loor. 2009. Peroxisome proliferator-activated receptor-gamma activation and long-chain fatty acids alter lipogenic gene networks in bovine mammary epithelial cells to various extents. J. Dairy Sci. 92:4276-4289. https://doi.org/10.3168/jds.2008-1932.

Latasa, M. J., Y. S. Moon, K. H. Kim, and H. S. Sul. 2000. Nutritional regulation of the fatty acid synthase promoter in vivo: Sterol regulatory element binding protein functions through an upstream region containing a sterol regulatory element. Proc. Natl. Acad. Sci. USA 97:10619-10624. https://doi.org/10.1073/pnas.180306597.

Li, J., J. Luo, H. Xu, M. Wang, J. Zhu, H. Shi, A. B. Haile, H. Wang, and Y. Sun. 2015. Fatty acid synthase promoter: characterization, and transcriptional regulation by sterol regulatory element binding protein-1 in goat mammary epithelial cells. Gene 561:157-164. https://doi.org/10.1016/j.gene.2015.02.034.

Li, N., F. Zhao, C. Wei, M. Liang, N. Zhang, C. Wang, Q. Z. Li, and X. J. Gao. 2014. Function of SREBP1 in the milk fat synthesis of dairy cow mammary epithelial cells. Int. J. Mol. Sci. 15:1699817013. https://doi.org/10.3390/ijms150916998.

Loor, J. J., M. Bionaz, and J. K. Drackley. 2013. Systems physiology in dairy cattle: Nutritional genomics and beyond. Annu. Rev. Anim. Biosci. 1:365-392. https://doi.org/10.1146/annurev-animal $-031412-103728$.

Ma, L., and B. A. Corl. 2012. Transcriptional regulation of lipid synthesis in bovine mammary epithelial cells by sterol regulatory element binding protein-1. J. Dairy Sci. 95:3743-3755. https://doi .org/10.3168/jds.2011-5083.

Magaña, M. M., S. H. Koo, H. C. Towle, and T. F. Osborne. 2000. Different sterol regulatory element-binding protein-1 isoforms utilize 
distinct co-regulatory factors to activate the promoter for fatty acid synthase. J. Biol. Chem. 275:4726-4733.

Moon, Y. A. 2017. The SCAP/SREBP pathway: A mediator of hepatic steatosis. Endocrinol. Metab. (Seoul) 32:6-10. https://doi .org/10.3803/EnM.2017.32.1.6.

Moon, Y. A., G. Liang, X. Xie, M. Frank-Kamenetsky, K. Fitzgerald, V. Koteliansky, M. S. Brown, J. L. Goldstein, and J. D. Horton. 2012. The Scap/SREBP pathway is essential for developing diabetic fatty liver and carbohydrate-induced hypertriglyceridemia in animals. Cell Metab. 15:240-246. https://doi.org/10.1016/j.cmet .2011.12.017.

Moon, Y. S., M. J. Latasa, M. J. Griffin, and H. S. Sul. 2002. Suppression of fatty acid synthase promoter by polyunsaturated fatty acids. J. Lipid Res. 43:691-698.

Ordovás, L., R. Roy, S. Pampin, P. Zaragoza, R. Osta, J. C. Rodriguez-Rey, and C. Rodellar. 2008. The g.763G $>$ C SNP of the bovine FASN gene affects its promoter activity via Sp-mediated regulation: Implications for the bovine lactating mammary gland. Physiol. Genomics 34:144-148. https://doi.org/10.1152/ physiolgenomics.00043.2008.

Oskouian, B., V. S. Rangan, and S. Smith. 1997. Regulatory elements in the first intron of the rat fatty acid synthase gene. Biochem. J. $324: 113-121$.

Osorio, J. S., J. Lohakare, and M. Bionaz. 2016. Biosynthesis of milk fat, protein, and lactose: Roles of transcriptional and posttranscriptional regulation. Physiol. Genomics 48:231-256. https://doi .org/10.1152/physiolgenomics.00016.2015.

Rawson, R. B., R. DeBose-Boyd, J. L. Goldstein, and M. S. Brown. 1999. Failure to cleave sterol regulatory element-binding proteins (SREBPs) causes cholesterol auxotrophy in Chinese hamster ovary cells with genetic absence of SREBP cleavage-activating protein. J. Biol. Chem. 274:28549-28556.

Reed, B. D., A. E. Charos, A. M. Szekely, S. M. Weissman, and M. Snyder. 2008. Genome-wide occupancy of SREBP1 and its partners NFY and SP1 reveals novel functional roles and combinatorial regulation of distinct classes of genes. PLoS Genet. 4:e1000133. https://doi.org/10.1371/journal.pgen.1000133.

Rome, S., V. Lecomte, E. Meugnier, J. Rieusset, C. Debard, V. Euthine, H. Vidal, and E. Lefai. 2008. Microarray analyses of SREBP-1a and SREBP-1c target genes identify new regulatory pathways in muscle. Physiol. Genomics 34:327-337. https://doi .org/10.1152/physiolgenomics.90211.2008

Roy, R., L. Ordovas, P. Zaragoza, A. Romero, C. Moreno, J. Altarriba, and C. Rodellar. 2006. Association of polymorphisms in the bovine FASN gene with milk-fat content. Anim. Genet. 37:215-218. https://doi.org/10.1111/j.1365-2052.2006.01434.x.

Roy, R., S. Taourit, P. Zaragoza, A. Eggen, and C. Rodellar. 2005. Genomic structure and alternative transcript of bovine fatty acid synthase gene (FASN): comparative analysis of the FASN gene between monogastric and ruminant species. Cytogenet. Genome Res. 111:65-73. https://doi.org/10.1159/000085672.
Rudolph, M. C., J. Monks, V. Burns, M. Phistry, R. Marians, M. R. Foote, D. E. Bauman, S. M. Anderson, and M. C. Neville. 2010. Sterol regulatory element binding protein and dietary lipid regulation of fatty acid synthesis in the mammary epithelium. Am. J. Physiol. Endocrinol. Metab. 299:E918-E927. https://doi.org/10 .1152/ajpendo.00376.2010.

Sato, R. 2009. SREBPs: Protein interaction and SREBPs. FEBS J. 276:622-627. https://doi.org/10.1111/j.1742-4658.2008.06807.x.

Seo, Y. K., H. K. Chong, A. M. Infante, S. S. Im, X. Xie, and T. F. Osborne. 2009. Genome-wide analysis of SREBP-1 binding in mouse liver chromatin reveals a preference for promoter proximal binding to a new motif. Proc. Natl. Acad. Sci. USA 106:13765-13769. https://doi.org/10.1073/pnas.0904246106.

Suburu, J., L. Shi, J. Wu, S. Wang, M. Samuel, M. J. Thomas, N. D. Kock, G. Yang, S. Kridel, and Y. Q. Chen. 2014. Fatty acid synthase is required for mammary gland development and milk production during lactation. Am. J. Physiol. Endocrinol. Metab. 306:E1132-E1143. https://doi.org/10.1152/ajpendo.00514.2013.

Xu, H., J. Luo, G. Ma, X. Zhang, D. Yao, M. Li, and J. J. Loor 2018. Acyl-CoA synthetase short-chain family member 2 (ACSS2) is regulated by SREBP-1 and plays a role in fatty acid synthesis in caprine mammary epithelial cells. J. Cell. Physiol. 233:1005-1016. https://doi.org/10.1002/jcp.25954.

Xu, H. F., J. Luo, H. P. Wang, H. Wang, T. Y. Zhang, H. B. Tian, D. W. Yao, and J. J. Loor. 2016a. Sterol regulatory element binding protein-1 (SREBP-1)c promoter: Characterization and transcriptional regulation by mature SREBP-1 and liver X receptor alpha in goat mammary epithelial cells. J. Dairy Sci. 99:1595-1604. https://doi.org/10.3168/jds.2015-10353.

Xu, H. F., J. Luo, W. S. Zhao, Y. C. Yang, H. B. Tian, H. B. Shi, and M. Bionaz. 2016b. Overexpression of SREBP1 (sterol regulatory element binding protein 1) promotes de novo fatty acid synthesis and triacylglycerol accumulation in goat mammary epithelial cells. J. Dairy Sci. 99:783-795. https://doi.org/10.3168/jds.2015-9736.

Yang, T., J. L. Goldstein, and M. S. Brown. 2000. Overexpression of membrane domain of SCAP prevents sterols from inhibiting SCAP.SREBP exit from endoplasmic reticulum. J. Biol. Chem. 275:29881-29886. https://doi.org/10.1074/jbc.M005439200.

Yao, D., J. Luo, Q. He, H. Shi, J. Li, H. Wang, H. Xu, Z. Chen, Y. Yi, and J. J. Loor. 2017. SCD1 alters long-chain fatty acid (LCFA) composition and its expression is directly regulated by SREBP-1 and PPARgamma 1 in dairy goat mammary cells. J. Cell. Physiol. 232:635-649. https://doi.org/10.1002/jcp.25469.

Zhu, J. J., J. Luo, Y. T. Sun, H. B. Shi, J. Li, M. Wu, K. Yu, A. B. Haile, and J. J. Loor. 2015. Short communication: Effect of inhibition of fatty acid synthase on triglyceride accumulation and effect on lipid metabolism genes in goat mammary epithelial cells. J. Dairy Sci. 98:3485-3491. https://doi.org/10.3168/jds.2014-8202. 